\title{
Original article (short paper) \\ Resistance exercise improves metabolic parameters and changes adipocyte-derived leptin: a comparison between genders in untrained adults
}

\author{
Gustavo Ribeiro da Mota \\ Universidade Federal do Triângulo Mineiro, Uberaba, MG, Brasil \\ Fábio Lera Orsatti \\ Universidade Federal do Triângulo Mineiro, Uberaba, MG, Brasil \\ Maria Andréia Delbin \\ Universidade de Campinas, Campinas, SP, Brasil \\ Angelina Zanesco \\ Universidade Estadual Paulista, Rio Claro, SP, Brasil
}

\begin{abstract}
This study examined gender differences after resistance exercise (RE) by measuring fasting plasma levels of creatine kinase, lipid profile, blood glucose, adiponectin, tumor necrosis factor alpha (TNF- $\alpha$ ), and leptin. Thirteen women ( $23.6 \pm 7.3$ years) and 11 men ( $29.5 \pm 9$ years) were enrolled in the study. Two bouts of RE were performed on two different occasions separated by $48 \mathrm{~h}$ each. Blood samples were collected and analyzed at baseline and $15 \mathrm{~h}$ after the last RE session. Increased creatine kinase levels and improvements in lipid profile and blood glucose were found for both genders. No changes in adiponectin and TNF- $\alpha$ levels were observed for both genders, but leptin levels were reduced $(p<0.05)$ only for women after RE. Collectively, our findings clearly show that RE was effective in lowering blood glucose and that this effect was not accompanied by changes in adiponectin levels in healthy subjects, indicating that RE is still an important tool for the prevention of metabolic diseases. Furthermore, two sessions of RE promoted a reduction in leptin levels in women, even though no changes in body weight were found, showing that RE is an interesting approach to study obese patients and metabolic regulation.
\end{abstract}

Keywords: resistance training; adiponectin; leptin; health; gender

\section{Introduction}

A healthy lifestyle has been strongly associated with the practice of regular physical activity, and evidence has shown that physically active subjects have greater longevity with a reduction in morbidity and mortality ${ }^{1}$. Aerobic exercise prevents and reduces the deleterious effects of several chronic diseases ${ }^{2}$.

Resistance exercise (RE) is associated with an increase in muscle strength, power, and rate of force development, consequently improving performance in daily activities and quality of life ${ }^{3}$. More recently, studies have shown that RE can also prevent diseases, such as obesity, diabetes, dyslipidemia, and hypertension, mainly in elderly and sedentary people ${ }^{3,4}$. On the other hand, it has been reported that molecular and cellular abnormalities associated with the development of obesity and insulin resistance are initiated in the youth. ${ }^{5}$ Indeed, there is an association between an early onset of physical activity practice and a lower occurrence of endocrine and cardiovascular diseases in adulthood ${ }^{6}$.

In this context, adipose tissue has been considered as an endocrine organ releasing several substancesincluding leptin, adiponectin, and tumor necrosis factor alpha (TNF- $\alpha)^{7}$. These adipocyte-derived cytokines influence a range of physiological functions, such as energy homeostasis, glucose regulation, and insulin sensitivity ${ }^{8}$. Some studies have evaluated the effect of aerobic exercises on adipocyte-derived cytokines in different subjects9. However, there are few studies investigating the gender-specific responses of blood glucose and lipid profile to RE and their possible association with cytokines in healthy individuals. Thus, the aim of this study was to evaluate gender-based differences in healthy, untrained subjects after two bouts of RE in relation to fasting plasma levels of creatine kinase (CK), lipid profile, blood glucose, adiponectin, tumor necrosis factor alpha (TNF- $\alpha$ ), and leptin.

\section{Methods}

\section{Participants}

This study was approved by the Ethics Committee Board of the University of Sao Paulo State (UNESP) and performed in accordance with international ethical standards. All participants signed an informed consent form. The subjects were 
recruited by advertisement and the inclusion criteria for participating were as follows: a) No previous engagement in RE programs, b) physically inactive, c) non-smoking, d) nondiabetic (fasting glucose level $<126 \mathrm{mg} / \mathrm{dL}$ ), e) normotensive, f) BMI $<29.9 \mathrm{~kg} / \mathrm{m}^{2}$, g) no intake of dietary supplements or potential ergogenic compounds of any kind, h) maintaining a stable weight for at least 6 months before the study, i) having no medical impediment to performance of RE, j) alcohol consumption and k) pregnancy. Twenty-four participants: 13 women (23.6 \pm 7.3 years) and 11 men ( $29.5 \pm 9$ years) fulfilled the inclusion criteria. Subjects were instructed to maintain their usual nutrition habits throughout the study period. Furthermore, they were asked to complete dietary records for all 3 days prior to blood collection. Alcohol and caffeine were prohibited during the week of the experiments.

\section{Protocol design}

Before starting the RE protocol, all participants were familiarized with the types of exercise and 12 repetition maximum (12-RM) test. The familiarization period lasted for six sessions for each participant. The RE protocol was monitored by the researchers and consisted of nine exercises (eight exercises quoted in the 12-RM test, more abdominal - without external load). The two bouts of RE were performed at the same time of the day (05:00 p.m.) to avoid circadian variation. Three sets of 12-RM with 1.5-2 min of rest between sets, and 2-5 min of rest between exercises were performed. Each session lasted nearly $1 \mathrm{~h}$. Total absolute load (sum of load $[\mathrm{kg}]$ used in the 12-RM test for the eight exercises) and total relative load (total absolute load $[\mathrm{kg}]$ divided per muscle mass $[\mathrm{mm}]-\mathrm{kg} / \mathrm{mm}$ ) were determined for of each individual. Two bouts of RE were performed on separate occasions $48 \mathrm{~h}$ apart. The protocol design is illustrated in Figure 1.

\section{2-RM strength test}

$12 \mathrm{RM}$ was defined as the maximum amount of load that could be lifted through a full range motion 12 times, as described previously ${ }^{10}$. Each subject performed a 12-RM strength test on eight exercises using resistance machines (Reforce ${ }^{\circledR}$ ): (I) $45^{\circ}$ leg press, (II) lat pull-down, (III) chest press, (IV) leg flexion, (V) leg extension, (VI) standing freeweight biceps curl, (VII) triceps pulley and (VIII) dumbbell lateral side shoulder raise. After a warm-up with low weight, the subject performed exercise beginning with $70 \%$ of their perceived $12 \mathrm{RM}$ for 12 repetitions. The weight was progressively increased by approximately $5-10 \mathrm{~kg}$ after each successful attempt until the $12 \mathrm{RM}$ was reached. A rest interval of 2 min was given between attempts and 5 min between exercises. The 12-RM values were then used to determine the corresponding load for the RE sessions. Movement velocity was controlled (approximately $3 \mathrm{~s}$ each repetition) through a pacer alarm $\left(D^{\prime}\right.$ Accord $\left.{ }^{\circledR}\right)$. All tests were supervised by the same investigator.

\section{Anthropometric measurements}

Both BMI and percentage of body fat $(\% \mathrm{BF})$ were calculated for all participants. Body density was estimated using the three skin fold site method according to a previous study ${ }^{11}$ and $\mathrm{BF} \%$ was predicted by the equation of Brozek and coworkers ${ }^{12}$.

\section{Blood sample collection}

Blood samples were taken before and after the protocol design after a 12-h overnight fast. Venous blood samples were collected using two vacuum tubes (BD vacutainer tubes ${ }^{\circledR}$ ): One for plasma (EDTA K3) and another for serum (clot activator and gel for serum separation). Plasma tubes were immediately centrifuged $(8000 \mathrm{~g})$ for $10 \mathrm{~min}$. Serum tubes sat for $30 \mathrm{~min}$ at room temperature and then centrifuged. After that, the supernatant (plasma and serum) was stored in aliquots at $-80^{\circ} \mathrm{C}$ for future biochemical assays.

\section{Biochemical assays}

Total cholesterol, high-density lipoprotein cholesterol (HDL-C), low-density lipoprotein cholesterol (LDL-C), triglycerides, and blood glucose were determined using automated standard methods (Cobas Mira Plus).

Adiponectin, TNF- $\alpha$, and leptin levels were quantified by enzyme-linked immunosorbent assay (Cay006Dan Chemical, Ann Arbor, MI).

Serum creatine kinase levels were measured enzymatically using commercial kits. Blood lactate concentration ([Lac]) was determined at rest and $3 \mathrm{~min}$ after the second RE session using a lactate analyzer (Accutrend-Roche Diagnostics ${ }^{\circledR}$, Germany).

All biochemical parameters, except [Lac], were assessed in duplicate.

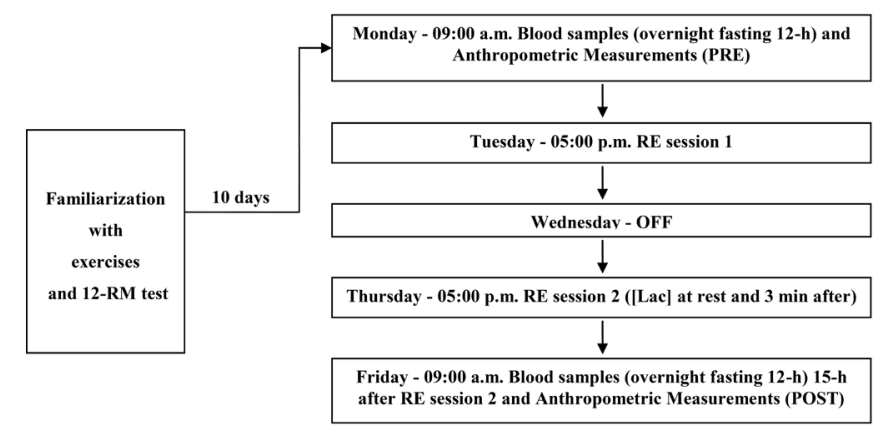

Figure 1. Experimental design. (12-RM $=12$ repetition maximum; $\mathrm{RE}=$ resistance exercise; $[\mathrm{Lac}]=$ blood lactate concentration) .

\section{Statistical analysis}

Data were analyzed by two-way (group x time) ANOVA for repeated measures to compare differences between genders as well as pre- and post-intervention. Pearson's coefficient was also employed to assess correlations. The significance level was set at $P<0.05$ (StatSoft, Inc., Tulsa, OK, USA). 


\section{Results}

Women presented higher body fat $(26.9 \pm 6 \%)$ than men $(16.7 \pm 3.5 \%)$, whereas greater BMI values were found for men $\left(26.2 \pm 2.7 \mathrm{~kg} . \mathrm{m}^{2}\right)$ than for women $\left(22.4 \pm 3.4 \mathrm{~kg} \cdot \mathrm{m}^{2}\right)$. No differences in dietary nutrient composition were found between the two groups ( $\%$ fat, carbohydrate, protein; $P>0.05$, data not shown).

\section{Total load performed and blood lactate}

The total absolute load used in RE sessions was significantly greater $(P<0.05)$ for men $(352.8 \pm 85.5 \mathrm{~kg})$ than for women $(185.7 \pm 46.8 \mathrm{~kg})$, whereas total relative load was not different $(P>0.05)$ between genders (men: $5.1 \pm 1 \mathrm{~kg} / \mathrm{mm}$; women: 4.4 $\pm 1.1 \mathrm{~kg} / \mathrm{mm}$ ). A significant increase in blood lactate levels was found after RE for both men (basal: $1.3 \pm 0.2$ and after RE: 7.5 $\pm 1.1 \mathrm{mmol} . \mathrm{L}^{-1}$ ) and women (basal: $1.2 \pm 0.2$ and after RE: 5.9 \pm 0.7 mmol. $\left.\mathrm{L}^{-1}\right)$.

\section{Creatine kinase (CK)}

Using RCV analyses, we observed that 9 of the 11 men displayed significantly increased CK levels after RE (152 \pm 144 to $\left.728 \pm 910 \mathrm{U}^{\mathrm{L}} \mathrm{L}^{-1}\right)$, whereas all women presented an increase in this parameter $(51 \pm 13$ to $1192 \pm 1460$ U.L.-1).

\section{Lipid profile and blood glucose}

In order to examine the effects of RE on lipid profile by gender, the difference in lipid profile before and after RE sessions was determined. For men, a significant decrease in total cholesterol and LDL-C was observed, whereas HDL-C was significantly increased after two sessions of RE. No significant changes were observed for triglycerides in men. Data are summarized in Table 1. Similarly, RE was effective in lowering total cholesterol and HDL-C in women. HDL-C was also significantly increased after the resistance protocol in women. Data are summarized in Table 2.

Regarding blood glucose, two sessions of RE significantly reduced blood glucose (approximately $-40 \%$ ) for both groups (99.9 \pm 9 to $60 \pm 6 \mathrm{mg} / \mathrm{dL}$ in women and $105 \pm 9$ to $62 \pm 4.7 \mathrm{mg} / \mathrm{dL}$ in men).

Table 1. Lipid profile evaluated individually before and after two sessions of resistance exercise in men $(\mathrm{n}=11)$.

\begin{tabular}{|c|c|c|c|c|}
\hline Biomarker & Subject & Measured values & latter-former $(\Delta)$ & $\%$ Change \\
\hline Total & 2 & $3-4.6$ & -1.6 & $-35 \%$ \\
\hline cholesterol & 5 & $2.2-2.9$ & -0.7 & $-24 \%$ \\
\hline $\mathrm{RCV}_{95 \%}=18.6 \%$ & 10 & $3.2-4$ & -0.8 & $-21 \%$ \\
\hline \multirow[t]{2}{*}{$\mathrm{RI}<5.18 \mathrm{mmol} / \mathrm{L}$} & 11 & $3.1-4$ & -0.9 & $-22 \%$ \\
\hline & 4 & $0.9-1.3$ & -0.4 & $-34 \%$ \\
\hline HDL cholesterol & 6 & $1.1-0.9$ & 0.2 & $+24 \%$ \\
\hline $\mathrm{RCV}_{95 \%}=22 \%$ & 7 & $1.4-0.9$ & 0.5 & $+49 \%$ \\
\hline \multirow[t]{3}{*}{$\mathrm{RI}>1.3 \mathrm{mmol} / \mathrm{L}$} & 8 & $0.9-0.6$ & 0.3 & $+47 \%$ \\
\hline & 11 & $1.0-0.5$ & 0.4 & $+89 \%$ \\
\hline & 2 & $1.6-2.9$ & -1.3 & $-45 \%$ \\
\hline LDL Cholesterol & 3 & $2.8-1.9$ & 0.9 & $+46 \%$ \\
\hline $\mathrm{RCV}=25.7 \%$ & 5 & $1.0-1.7$ & -0.8 & $-44 \%$ \\
\hline \multirow[t]{2}{*}{$\mathrm{RI}<3.37 \mathrm{mmol} / \mathrm{L}$} & 10 & $2.1-3.3$ & -1.2 & $-36 \%$ \\
\hline & 11 & $1.9-3.3$ & -1.3 & $-41 \%$ \\
\hline
\end{tabular}

$\mathrm{RI}=$ Reference interval; $\Delta=$ difference between two consecutive analyses.

Table 2. Lipid profile evaluated individually before and after two sessions of resistance exercise in women $(\mathrm{n}=13)$.

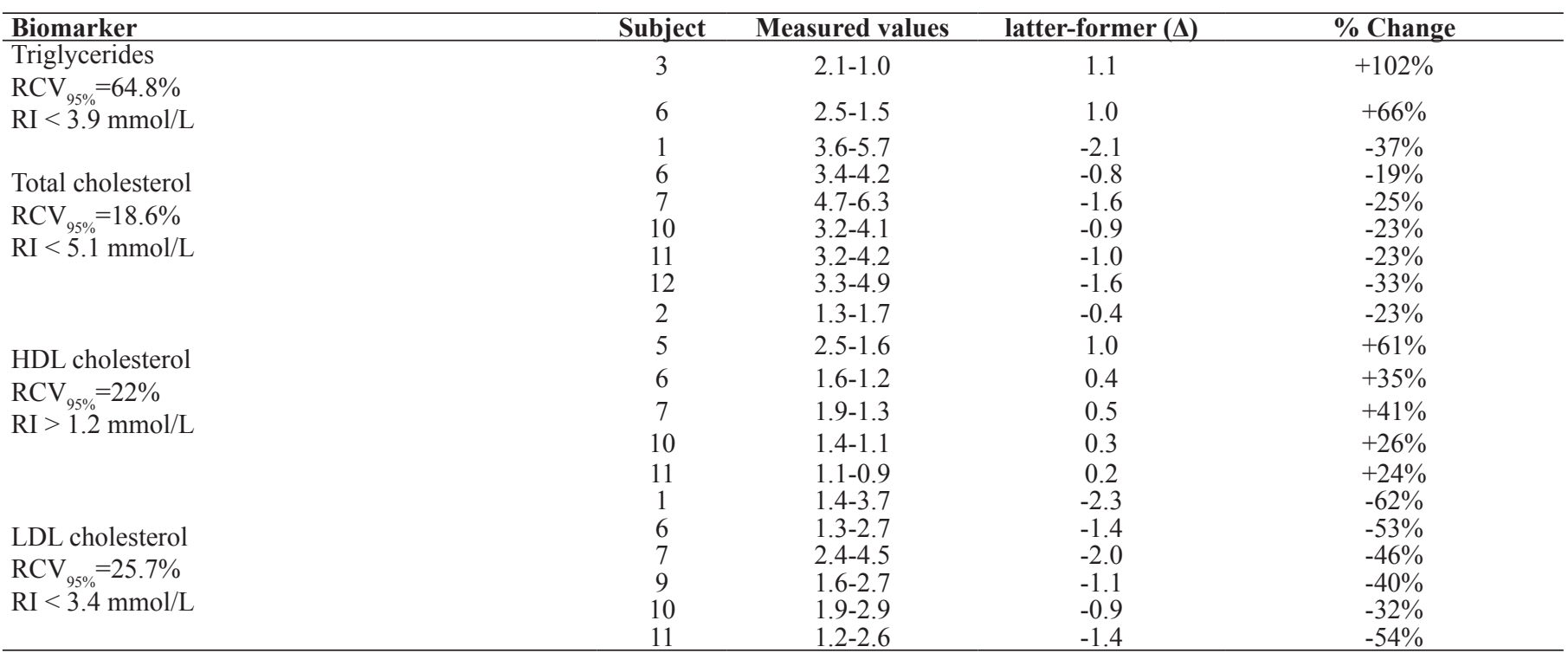

$\mathrm{RI}=$ Reference interval; $\Delta=$ difference between two consecutive analyses. 


\section{Pro- and anti-inflammatory mediators}

At basal conditions, it was observed that both leptin and adiponectin levels were significantly higher, nearly $200 \%$ and $135 \%$, respectively, in women as compared with men. Two sessions of RE failed to cause any alterations in adiponectin levels in both groups, whereas leptin concentration was significantly reduced in women who performed the RE sessions. Regarding the cytokine TNF- $\alpha$, no gender differences were observed for this pro-inflammatory mediator. In addition, two sessions of RE did not affect this biomarker in either groups. Data are summarized in Table 3.

The correlation analysis showed a positive relationship between leptin levels and $\mathrm{BF} \%$ for both genders together $(r=$ 0.82 ) or separately ( $r=0.7$ for women and $r=0.76$ for men).

Table 3. Effects of two bouts of resistance exercise on adiponectin, leptin, and TNF- $\alpha$ levels in healthy women and men.

\begin{tabular}{ccc}
\hline Biomarker & $\begin{array}{c}\text { Men } \\
(\mathbf{n}=\mathbf{1 1})\end{array}$ & $\begin{array}{c}\text { Women } \\
(\mathbf{n}=\mathbf{1 3})\end{array}$ \\
\hline Adiponectin $\left(\boldsymbol{\mu g} \cdot \mathbf{m L}^{-1}\right)$ & & \\
Pre & $3.7 \pm 1.5^{\#}$ & $8.7 \pm 3$ \\
Post & $3.7 \pm 1.9^{\#}$ & $9.2 \pm 3.2$ \\
TNF- $\boldsymbol{\alpha}\left(\mathbf{n g} \cdot \mathbf{m L}^{-1}\right)$ & & \\
Pre & $88.8 \pm 6.7$ & $80.6 \pm 12.7$ \\
Post & $91.9 \pm 6.5$ & $84.3 \pm 15.6$ \\
Leptin (ng.mL $\left.{ }^{-1}\right)$ & & \\
Pre & $6.7 \pm 3.6 \#$ & $20.2 \pm 10.8$ \\
Post & $5.9 \pm 2.3 \#$ & $15.6 \pm 9.3^{*}$ \\
\hline
\end{tabular}

Data are mean \pm SD. ${ }^{*}$ difference between groups. * Difference between preand post- exercise resistance sessions.

\section{Discussion}

To our knowledge, this is the first study to verify the adipokine responses to a controlled RE protocol in healthy untrained subjects, though other related studies have investigated special populations, such as diabetics ${ }^{13}$, obese ${ }^{9}$, elderly ${ }^{14}$, and athletes ${ }^{15}$. Accordingly, the main findings of this report were a) the leptin levels of women are more responsive to RE than those of men b) RE is effective in lowering metabolic parameters in both genders, measured by lipid profile and blood glucose. Taken together these findings suggest that RE could be a tool for preventing chronic diseases in healthy adults.

Anthropometric parameters (BMI and \%BF) did not change after RE sessions due the short intervention time and unaltered nutrition habits throughout the study period. Therefore, they cannot explain the significant changes that occurred. Despite the similar relative intensity of the RE protocol between genders (i.e., 12-RM for both), men presented higher total absolute load when compared with women. This result was expected because both groups were untrained and it is in accordance with a previous study ${ }^{16}$.
For both genders [Lac] increased significantly after the RE protocol (women: approximately $386 \%$ and men: approximately $499 \%$ ) relative to the resting state. The [Lac] found here is commonly seen with RE schemes designed to stimulate muscle hypertrophy because there is a relationship between acute [Lac] response and anabolic hormones ${ }^{17}$, such as growth hormone $(\mathrm{GH})^{18}$. Additionally, the [Lac] observed illustrates a great contribution from the anaerobic lactic pathway to cover the energetic demand and it was probably due to time of muscle action in our RE protocol (approximately $36 \mathrm{~s}$ each set) and brief rest periods between sets (1.5 $\mathrm{min})$.

Serum CK activity is a marker of muscle damage ${ }^{19}$. Our results showed that for both genders there were a significant increment in CK levels. Because our volunteers were untrained, these results were expected and suggest that RE achieved the "break point of CK release" (i.e., two or three times higher than resting condition $)^{19}$. Despite the great individual variability commonly found in $\mathrm{CK}$, our data showed significant increases including from RCV analyses, which has been a better guide to interpreting changes in serial results ${ }^{20}$. Considering that increased CK levels occurred in both genders, the gender-based differences found in other parameters measured cannot be explained by muscle damage.

Plasma lipoproteins play an important role in the genesis of atherosclerosis and evidence has shown that aerobic exercise ameliorates lipid profile and consequently, protects against the atherosclerosis process ${ }^{21}$. On the other hand, studies evaluating the effects of RE on the lipid profile are not conclusive. In fact, it was demonstrated that RE promotes an improvement in lipid profile $^{22}$ or causes no change ${ }^{23}$. Our findings showed an improvement in lipid profile in response to acute RE in both genders, as evidenced by RCV analyses. This improvement seems to have occurred independently of inflammatory markers (TNF- $\alpha$ and adiponectin). Considering that $\mathrm{GH}$ has an important role in lipolysis and that high [Lac] is associated with the release of $\mathrm{GH}^{24}$, we could speculate that the acute improvement in lipid profile could be related to high [Lac] caused by our RE protocol.

Our protocol markedly reduced glucose concentration after two bouts of RE in both groups. These reductions are likely related to glycogen depletion, because our RE protocol used large muscle mass and we had great anaerobic participation (high $[\mathrm{Lac}])^{25}$. In addition, it is well known that physical exercise, aerobic or resistance, increases insulin sensitivity, increasing glucose uptake in skeletal muscle, either in healthy subjects or diabetic patients ${ }^{26}$. In fact, it was recently demonstrated that muscular strength was positively associated with protection against cardiovascular disease in $\operatorname{men}^{27}$. Thus, our study reinforces the importance of RE in preventing chronic diseases such as type 2 diabetes.

As expected, adiponectin levels were higher in women than in men at baseline and two sessions of RE failed to change the levels this adipokine. Evidence has shown that physical exercise with high energy expenditure promotes beneficial effects by decreasing leptin and increasing adiponectin concentrations in diabetic $^{28}$, obese ${ }^{9}$, and elderly subjects ${ }^{14}$. Considering that we examined healthy subjects, the lack of changes in adiponectin might have been due to the absence of metabolic disorders. In 
contrast, the RE protocol reduced leptin levels in women but not in men, showing that this adipocyte-derived adipokine is more responsive to RE in women than in men. Accordingly, a recent review about $\mathrm{RE}$ and adipokines also confirms that women are more responsive than men in relation to reduction after exercise training, but the mechanisms behind are not yet clear $^{29}$. Regarding TNF- $\alpha$, no differences were found at baseline and RE failed to cause any changes in this proinflammatory mediator. TNF- $\alpha$ is considered a key molecule in peripheral insulin resistance and longitudinal as well as cross-sectional studies have shown the inhibitory effects of physical exercise on this proinflammatory biomarker ${ }^{26,30}$. However, most studies were conducted in patients with metabolic disorders or elderly patients. In addition, our study is the first to examine gender comparisons using an RE protocol. The relationship between leptin levels and body fat is well established ${ }^{15}$ and in agreement with previous studies, we found a positive relationship in both genders $(r=0.82)$.

Collectively, our findings clearly show that RE was effective in lowering blood glucose and that this effect was not accompanied by changes in adiponectin levels in healthy subjects, indicating that RE still an important tool to prevent metabolic diseases. Furthermore, two sessions of RE promoted a reduction in leptin levels in women, even though no changes in body weight were found, showing that RE is an interesting approach to studying obese patients and metabolic regulation.

\section{References}

1. Zanesco A, Antunes E. Effects of exercise training on the cardiovascular system: pharmacological approaches. Pharmacol Ther. 2007; 114(3):307-317.

2. Mota CSA, Mello MAR. Exercise and metabolic syndrome. Motriz J Physical Education. 2006; 12(2):185-193.

3. Phillips SM. Resistance exercise: good for more than just Grandma and Grandpa's muscles. Appl Physiol Nutr Metab. 2007; 32(6):1198-1205.

4. Xiao T, Fu YF. Resistance training vs. aerobic training and role of other factors on the exercise effects on visceral fat. Eur Rev Med Pharmacol Sci. 2015; 19(10):1779-84.

5. Dwyer T, Magnussen CG, Schmidt MD, Ukoumunne OC, Ponsonby AL, Raitakari OT, et al. Decline in physical fitness from childhood to adulthood associated with increased obesity and insulin resistance in adults. Diabetes Care. 2009; 32(4):683-687.

6. Fernandes RA, Zanesco A. Early physical activity promotes lower prevalence of chronic diseases in adulthood. Hypertens Res. 2010; 33(9):926-931.

7. Vazquez-Vela ME, Torres N, Tovar AR. White adipose tissue as endocrine organ and its role in obesity. Arch Med Res. 2008; 39(8):715-728.

8. Kotani K, Sakane N, Saiga K, Sano Y, Kurozawa Y. Lifestylerelated determinants and serum adiponectin concentrations in a general population of Japanese females. Arch Med Res. 2007; 38(8):887-890.
9. Kondo T, Kobayashi I, Murakami M. Effect of exercise on circulating adipokine levels in obese young women. Endocr J. 2006; 53(2):189-195.

10. Ortego AR, Dantzler DK, Zaloudek A, Tanner J, Khan T, Panwar $\mathrm{R}$, et al. Effects of gender on physiological responses to strenuous circuit resistance exercise and recovery. J Strength Cond Res. 2009; 23(3):932-938.

11. Jackson AS, Pollock ML. Generalized equations for predicting body density of men. Br J Nutr. 1978; 40(3):497-504.

12. Brozek J, Grande F, Anderson JT, Keys A. Densitometric Analysis of Body Composition: Revision of Some Quantitative Assumptions. Ann N Y Acad Sci. 1963; 110:113-140.

13. Kanaley JA, Fenicchia LM, Miller CS, Ploutz-Synder LL, Weinstock RS, Carhart R, Azevedo JLJr. Resting leptin responses to acute and chronic resistance training in type 2 diabetic men and women. Int J Obes Relat Metab Disord. 2001; 25(10):1474-1480.

14. Fatouros IG, Tournis S, Leontsini D, Jamurtas AZ, Sxina M, Thomakos $\mathrm{P}$, et al. Leptin and adiponectin responses in overweight inactive elderly following resistance training and detraining are intensity related. J Clin Endocrinol Metab. 2005; 90(11):5970-5977.

15. Jurimae J, Jurimae T. Leptin responses to short term exercise in college level male rowers. Br J Sports Med. 2005; 39(1):6-9.

16. Miller, AE, MacDougall JD, Tarnopolsky MA, Sale DG. Gender differences in strength and muscle fiber characteristics. Eur J Appl Physiol Occup Physiol. 1993; 66(3):254-262.

17. Crewther B, Cronin J, Keogh J. Possible stimuli for strength and power adaptation : acute metabolic responses. Sports Med. 2006; 36(1):65-78.

18. Goto K, Ishii N, Kizuka T, Takamatsu K. The impact of metabolic stress on hormonal responses and muscular adaptations. Med Sci Sports Exerc. 2005 37(6):955-963.

19. Totsuka M, Nakaji S, Suzuki, K, Sugawara K, Sato K. Break point of serum creatine kinase release after endurance exercise. J Appl Physiol. 2002; 93(4): 1280-1286.

20. Ricos C, Cava F, Garcia-Lario JV, Hernandez A, Iglesias N, Jimenez CV, et al. The reference change value: a proposal to interpret laboratory reports in serial testing based on biological variation. Scand J Clin Lab Invest. 2004; 64(3): 175-184.

21. Banz WJ, Maher MA, Thompson WG, Bassett DR, Moore W, Ashraf M, et al. Effects of resistance versus aerobic training on coronary artery disease risk factors. Exp Biol Med (Maywood). $2003 ; 228(4): 434-440$.

22. Hill S, Bermingham MA, Knight PK. Lipid metabolism in young men after acute resistance exercise at two different intensities. J Sci Med Sport. 2005; 8(4):441-445.

23. Nikolaidis MG, Paschalis V, Giakas G, Fatouros IG, Sakellariou GK, Theodorou AA, et al. Favorable and prolonged changes in blood lipid profile after muscle-damaging exercise. Med Sci Sports Exerc. 2008; 40(8):1483-1489.

24. Godfrey RJ, Whyte GP, Buckley J, Quinlivan R. The role of lactate in the exercise-induced human growth hormone response: evidence from McArdle disease. Br J Sports Med. 2009; 43(7):521-525.

25. Christ-Roberts CY, Mandarino LJ. Glycogen synthase: key effect of exercise on insulin action. Exerc Sport Sci Rev. 2004; 32(3):90-94. 
26. Knudsen SH, Pedersen BK. Targeting Inflammation Through a Physical Active Lifestyle and Pharmaceuticals for the Treatment of Type 2 Diabetes. Curr Diab Rep. 2015; 15(10):82.

27. Ruiz JR, Sui X, Lobelo F, Morrow JR, Jr, Jackson AW, Sjostrom $\mathrm{M}$, Blair SN. Association between muscular strength and mortality in men: prospective cohort study. Bmj. 2008: 337:a439.

28. Bluher M, Bullen JW, Jr, Lee JH, Kralisch S, Fasshauer M, Kloting $\mathrm{N}$, et al. Circulating adiponectin and expression of adiponectin receptors in human skeletal muscle: associations with metabolic parameters and insulin resistance and regulation by physical training. J Clin Endocrinol Metab. 2006. 91(6):2310-2316.

29. de Salles BF, Simao R, Fleck SJ, Dias I, Kraemer-Aguiar LG, Bouskela E. Effects of resistance training on cytokines. Int J Sports Med. 2010; 31(7): 441-450.

30. Ferguson MA, White LJ, McCoy S, Kim H.W, Petty T, Wilsey J. Plasma adiponectin response to acute exercise in healthy subjects. Eur J Appl Physiol. 2004; 91(2-3):324-329.

\section{Corresponding author}

Gustavo Ribeiro da Mota, PhD

Department of Sport Sciences, Federal University of Triângulo Mineiro (UFTM), Uberaba, MG, Brazil

Email: grmotta@gmail.com

Manuscript received on March 30, 2015

Manuscript accepted on April 10, 2016

\section{Acknowledgments}

The authors gratefully acknowledge all the subjects who participated in this study for their cooperation.

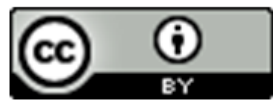

Motriz. The Journal of Physical Education. UNESP. Rio Claro, SP, Brazil - eISSN: 1980-6574 - under a license Creative Commons - Version 3.0 